\title{
The Grid Giant Star Survey for the Space Interferometry Mission
}

Richard J. Patterson, Steven R. Majewski, Catherine L. Slesnick, Jaehyon Rhee, Jeffrey D. Crane, Allyson A. Polak

Astronomy Department, University of Virginia, Charlottesville, VA 22903, USA

Arunav Kundu

Astronomy Department, Yale University, New Haven, CT 06520, USA

William E. Kunkel

Las Campanas Observatory, Casilla 601, La Serena, CHILE

Douglas Geisler, Ricardo Muñoz, Jose Arenas, Juan Seguel, Wolfgang Gieren

Departamento de Fisica, Universidad de Concepción, Concepción, CHILE

Verne V. Smith

Department of Physics, University of Texas, El Paso, TX 79968 USA

\section{G. Fritz Benedict}

McDonald Observatory, University of Texas, Austin, TX 78712 USA

Kathryn V. Johnston

Astronomy Department, Wesleyan University, Middleton, CT 06459 $U S A$

\begin{abstract}
NASA's Space Interferometry Mission (SIM), scheduled for launch in 2009, will determine the positions of thousands of stars as faint as $V=20$ to a precision better than 4 microarcseconds ( $\mu$ as). A key part of the mission is the Astrometric Grid, which is a reference frame of several thousand stars with $V \leq 13$ against which all relative measurements will be calibrated. To serve as a reliable inertial reference frame, the Grid must be astrometrically stable against photocenter jitter (from planets, binary companions, flaring or spotting) at the $\sim 4 \mu$ as level. Sub-solar metallicity giant stars, by virtue of their intrinsic luminosity, can probe the Galaxy to greater distances than almost any other stellar type at the same apparent magnitude. Thus, distant $(>3 \mathrm{kpc})$ giants with $V<13$ will have proportionately smaller astrometric jitter compared to other potential Astrometric Grid star candidates. The Grid Giant Star Survey is a patchwork all-sky survey to find sub-solar metallicity $\mathrm{K}$ giants
\end{abstract}


for the Grid, and to provide a unique database for studies of Galactic stellar populations. We describe here the survey characteristics and give examples of results to date.

\section{Introduction}

The Space Interferometry Mission (SIM) is a micro-arcsecond precision interferometer to be launched by NASA into earth-trailing orbit in 2009. SIM performs astrometry by measuring the additional path length taken by starlight to the slightly more distant of a pair of collecting apertures (located at the ends of a 10-m baseline). Absolute positions of stars over many degrees ("wide-angle mode") are obtained via comparison of angles from target stars to a well-defined grid of several thousand astrometric standard stars. Picometer accuracy in delay line measures translates to a single measure positional precision (for wide angle astrometry) of $10 \mu$ as. Observations between stars are repeated over the whole sky during the 5 year mission, resulting in mission-end precisions better than $4 \mu$ as. The complete list of reference star positions (including parallactic and proper motions) will constitute the mission Astrometric Grid. All SIM science programs requiring absolute astrometry will make use of this grid.

The high degree of astrometric precision required from the Grid places severe limits on the needed stability of individual Grid stars. A further requirement, that the majority of Grid stars have $V<13$ (with the exception of a relatively small sample of quasars for an extragalactic frame tie), arises from a desire to minimize the time spent on the Grid to less than $20 \%$ of the total mission time; the goal is to achieve the minimum mission fraction as set only by the time to acquire stars, with little contribution by integration time, and this is the case for stars with $V<13$. Grid candidates of all stellar types suffer to various degrees from the following sources of astrometric instability: 1) binary companions, 2) brown dwarfs, 3) planets, 4) photocenter wander from starspots and flares (SIM resolves the photospheres of $V<13$ stars). In principle, SIM itself can be used to detect astrometrically unstable stars in the Grid, but the entire mission will be jeopardized if too many stars drop out of the Grid. Thus, before launch it is critical to prepare an Astrometric Grid with stars for which the stability problems are minimized. This requires: a) a wise choice of stellar types for the Astrometric Grid, and b) an ambitious pre-launch observational campaign to weed out astrometrically unstable, problem stars.

We have been sponsored by NASA to conduct the Grid Giant Star Survey (GGSS) to find bright $(V<13)$, sub-solar metallicity giant stars for the SIM Astrometric Grid. Giant stars have been selected for the Grid because (1) they are common in all stellar populations and along all lines of sight, and (2) compared to almost any other class of star, giants at the same apparent magnitude will be more distant and therefore contribute a compensatory reduction in any astrometric jitter. To further decrease astrometric jitter, we search for sub-solar abundance $\mathrm{K}$ giants from the Galactic thick disk and halo with apparent magnitudes near the Astrometric Grid limit of $V \sim 13$. The net effects of extreme age and low metallicity increase the intrinsic brightness of giants, so that greater distances are attainable at the same apparent magnitude limit. We outline these 
and other advantages of sub-solar metallicity giants in $\S 2$. The details of the photometric technique and the logistics of the GGSS are detailed in $\S 3$.

Apart from their use in the SIM Astrometric Grid, stars from the GGSS will be useful for a host of Galactic structure studies, even before a subsample of the stars (those selected for the SIM Grid, and perhaps others submitted as SIM strategic targets) will have extremely precise, SIM-measured parallaxes and proper motions. We discuss the properties and some uses of the GGSS stars in $\S 4$.

\section{Population II Giant Stars as Grid Stars}

\subsection{Distance and Age}

Intermediate to Extreme Population II giant stars are excellent candidates for the All-Sky Astrometric Grid. A primary advantage is that at $0 \gtrsim M_{V} Z-3$, giants are at $\sim 1-10 \mathrm{kpc}$ for $V=9-12$. Searching to $V \sim 13$ ensures that we can find numerous giant stars from the Galactic Intermediate Population II and halo. It is now established that the Intermediate Population II is likely to be as old as the halo (Nissen \& Schuster 1991), and both populations contain members that are extremely metal-poor (Carney et al. 1996). With a judicious selection of targets from a thorough search, and pushing the mean magnitude of the sample to the Astrometric Grid limit of $V \sim 13$, a sample of giants with median distance of $\sim 3 \mathrm{kpc}$, and with at least $25 \%$ reaching to $>6 \mathrm{kpc}$, is being obtained.

Moreover, older stellar systems are less likely to have well established planetary systems, a possible source of astrometric jitter. Of course, inflated giant stars will not have an inner zone of terrestrial planets, but old, Population II giants are also less likely to have Jovian type planets, according to the standard notion that even giant gas planets begin life by accreting around rocky cores of ten Earth mass size (Stevenson 1985; Cameron 1988). The lack of observed planetary systems in the $[\mathrm{Fe} / \mathrm{H}]=-0.7$ globular cluster 47 Tuc (Gilliland et al. 2000) appears to strengthen this argument. Indeed, stars with known planetary systems are observed to be anomalously metal-rich when compared to stars of similar age (Gonzalez 1999).

\subsection{Binary Fraction}

As with most types of stars, little is known with certainty regarding the binary fraction in Population II giants. The binary fraction of Population II giant star progenitors is expected to be $\sim 30 \%$, roughly equivalent to that for Population I dwarfs (Duquennoy \& Mayor 1991, Ryan 1992). For example, low density globular clusters (where most primordial binaries would not have been tidally disrupted) appear to have a binary frequency close to that of Population I stars (Hut et al. 1992). For K giant stars in particular, the binary fraction for short period binaries is $\sim 15-20 \%$ for both Population I and II (Harris \& McClure 1983). Close binaries, those most likely to create significant photocenter movement around the barycenter in the SIM lifetime, will have likely been accreted during the red giant swelling phase of the primary. 


\subsection{Starspotting and Flaring}

Even at $10 \mathrm{kpc}$ the photosphere of a K3 III giant subtends an angle of 25 $\mu$ arcsecs, so starspotting and chromospheric activity (flaring) could affect SIM photocenters. However, rotationally induced chromospheric activity (as measured by CII and CIV emission line fluxes) is strongly correlated with $B-V$ color, and falls rapidly from a peak in line flux for early $G$ giants to very low levels for colors redder than $B-V \sim 0.9$ corresponding to late $\mathrm{G}$ or early $\mathrm{K}$ giants (Simon \& Drake 1989). Similar trends are seen in the CaII H and K lines. Gray (1989) finds that as giants evolve across the H-R diagram, surface rotation declines abruptly for types later than G3 III. This is thought to be caused by a dynamo-generated magnetic braking mechanism. In addition, few slowly rotating, later-type giants show signs of variability (Hall 1991). We therefore expect lower amounts of rotationally induced chromospheric activity and spotting in later-type giants.

Less evolved, solitary $\mathrm{G}$ and $\mathrm{K}$ subgiants that do exhibit activity are thought to be located near the base of the red giant branch as they enter a phase of stellar evolution where their convective zones encroach on their more rapidly rotating radiative cores, leading to dynamo driven stellar activity. These less evolved, less desirable grid candidate stars should be identifiable by their bluer colors.

In addition to any emission line flux that can be attributed to a magnetic dynamo driven by rotation, there also appears to be a "basal" component, present in all late-type stars (Rutten et al. 1991). This basal flux level is independent of the stellar metallicity (Cuntz, Rammacher \& Ulmschneider 1994), and it appears to indicate the onset of a hot outflow from stars near the tip of the red giant branch, at $M_{V}<-1.7$ (Dupree \& Smith 1995). This wind from the nonpulsating, inactive late-type giants is thought to be caused by acoustic shock heating of the chromosphere. Because this is a symmetric outflow from a slowly rotating, non-pulsating, inactive star, it should not affect the utility of halo $\mathrm{K}$ giants as astrometric reference stars.

In summary, astrometric jitter from binarity, flaring or starspotting should be no worse a problem for Population II giants than other types of stars, and giant stars, particularly late $\mathrm{G}$ and $\mathrm{K}$ type giants, may conceivably be better in this regard. In any case, several groups are monitoring variability in giant star brightness and radial velocity specifically to understand these issues as they pertain to the stability of SIM reference stars. The results of this work will provide an important check on the above claims.

\section{Observing Strategy and Status}

We (Majewski et al. 2000a) have developed, tested and begun using an efficient technique for finding giant stars photometrically. It is a variant of the combined Washington/DDO51 filter system by Geisler (1984) - simplified to a three filter system for the specific purpose of identifying distant giant stars - and relies on the gravity sensitivity of the $\mathrm{Mg} I$ triplet $+\mathrm{MgH}$ feature near $5150 \AA$ in $\mathrm{F}-\mathrm{K}$ stars (Figure 1). Imaging in the Washington $M$ and $T_{2}$ filters (Canterna 1976) provides a reliable measure of stellar $T_{\text {eff }}$, while the continuum subtracted flux in the $\mathrm{MgH}+\mathrm{Mgb}$ region is given by $M-D D O 51$. In Majewski et al. (2000a) we demonstrate the effectiveness of the $\left(M-T_{2}, M-D D O 51\right)$ diagram for 
separating dwarf and giant stars (Figure 2). With this filter system, we have nearly finished the photometric search phase of our southern GGSS (SGGSS) with the Swope 1-m telescope $(\S 3.1)$.

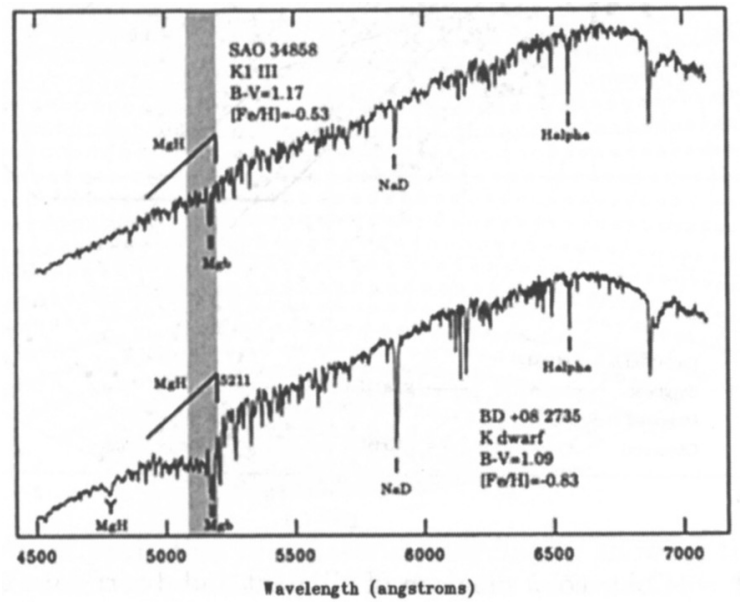

Figure 1. Comparison of spectra for $\mathrm{K}$ giant and dwarf stars of similar color and abundance, illustrating the dependence of the $\mathrm{MgH}+\mathrm{Mgb}$ triplet on luminosity class. The location of the DDO51 filter bandpass is indicated by the shaded region.

Targeted GGSS fields (or bricks) are shown in Figure 3, with the filled circles indicating the bricks that have been photometrically observed to date. Along each line of declination the bricks are separated by $7 .^{\circ} 06$ in RA. Declination bands are separated by $3 .^{\circ} 53$ in declination, and the brick/gap spacing along each declination band is staggered (brick centers shifted by $3 .^{\circ} 53$ in RA) with respect to those on the adjacent band. This results in a total of 1306 bricks on the celestial sphere, with a typical mean spacing between the bricks of $\sim 6^{\circ}$. With this density, there are about $6-7$ bricks to be found in each SIM $15^{\circ}$ diameter field of regard. Our goal is to find at least one good giant candidate in each brick, and thereby supply about 6-7 Grid stars per field of regard. To reach this goal, we are starting with a several times larger initial sample, from which we will pick the best candidate per brick after further study (§3.2-3.3).

\subsection{Photometric Imaging}

South of $\delta=+20^{\circ}$, we have used the Swope 1-m telescope at Las Campanas for the initial imaging, as well as the follow-up, low resolution spectroscopy. A SITe $2 \mathrm{~K} \times 2 \mathrm{~K}$ chip provides a $23 ! 7 \times 23 ! 7$ field of view at a plate scale of $0^{\prime \prime} 696 /$ pixel. To ensure that we obtain several bright, sub-solar metallicity giant candidates per brick, the southern GGSS bricks are constructed of sets of three adjacent and overlapping pointings of the Swope telescope, for a total of $1^{\circ} \times 0.33^{\circ}$ per brick.

In the north, we are using the 0.8-m telescope and Prime Focus Camera (PFC) at McDonald Observatory, with a field of view of $46.5 \times 46 ! 5$, and a plate scale of 1 ". $355 /$ pixel. The large PFC field of view has allowed us to construct 


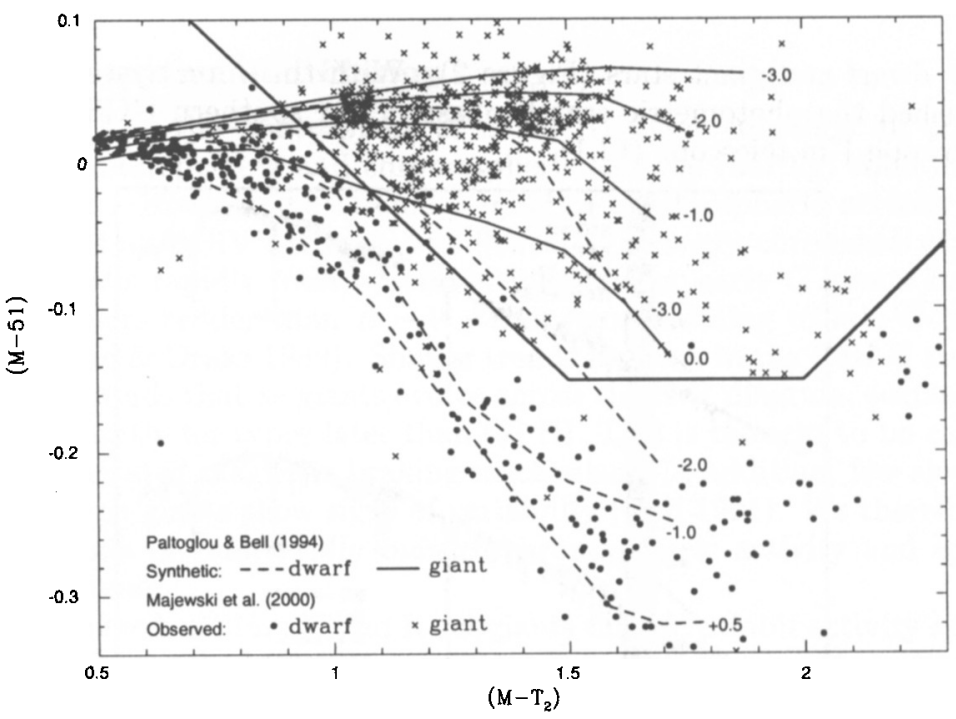

Figure 2. Color-color diagram of all giant and dwarf data in Majewski et al. (2000a), showing how our filter system separates giants from most dwarf stars for late $G$ - early $M$ spectral types. Data from 42 open and globular clusters and a sample of field dwarf and giant stars are shown. $X$ 's represent giant stars, while -'s represent dwarf stars. Giant stars are, for a large range of $\left(M-T_{2}\right)_{o}$ colors, well separated from the dwarfs, for all metallicities.

the northern bricks from a single telescope pointing. The coarse pixel scale does not affect our ability to identify the bright giants for the GGSS, and crowding does not appear to be a problem at $V<13$ even close to the Galactic plane.

We have completed imaging in 830 of the 1306 GGSS bricks (64\%). Imaging for the southern GGSS $\left(\delta<+20^{\circ}\right)$ is $80 \%$ complete (Figure 3 ).

\subsection{Low Resolution Spectroscopy}

Once $V<13$, sub-solar metallicity giant candidates are identified photometrically, we obtain follow-up, low-resolution ( $1 \AA /$ pixel) spectroscopy to verify them. A spectral range that we have found to be useful for spectroscopic dwarf/giant separation is $4800-6800 \AA$, which, of course, contains the gravity sensitive $\mathrm{MgH}$ band and $\mathrm{Mgb}$ triplet, but the gravity sensitive $\mathrm{Na} \mathrm{D}$ doublet as well. We are experimenting with various schemes to mask the spectra into three sets of lines with principal sensitivities to $\log g,[\mathrm{Fe} / \mathrm{H}]$, or $T_{\text {eff }}$, in order to give more precision in these quantities for our stars. With about $1 \AA /$ pixel scales, and $3-4 \AA$ resolution we also get $10-15 \mathrm{~km} / \mathrm{s}$ accuracy radial velocities, which provide useful information on the population membership of the stars. In the south, we have been using the Modular Spectrograph on the Swope 1-m for this work, and have obtained spectra of over 1000 giant candidates to date. For low resolution spectroscopy of the northern GGSS, we are building a fiber-fed bench spectrograph for the University of Virginia's Fan Mtn. 1-m telescope. 


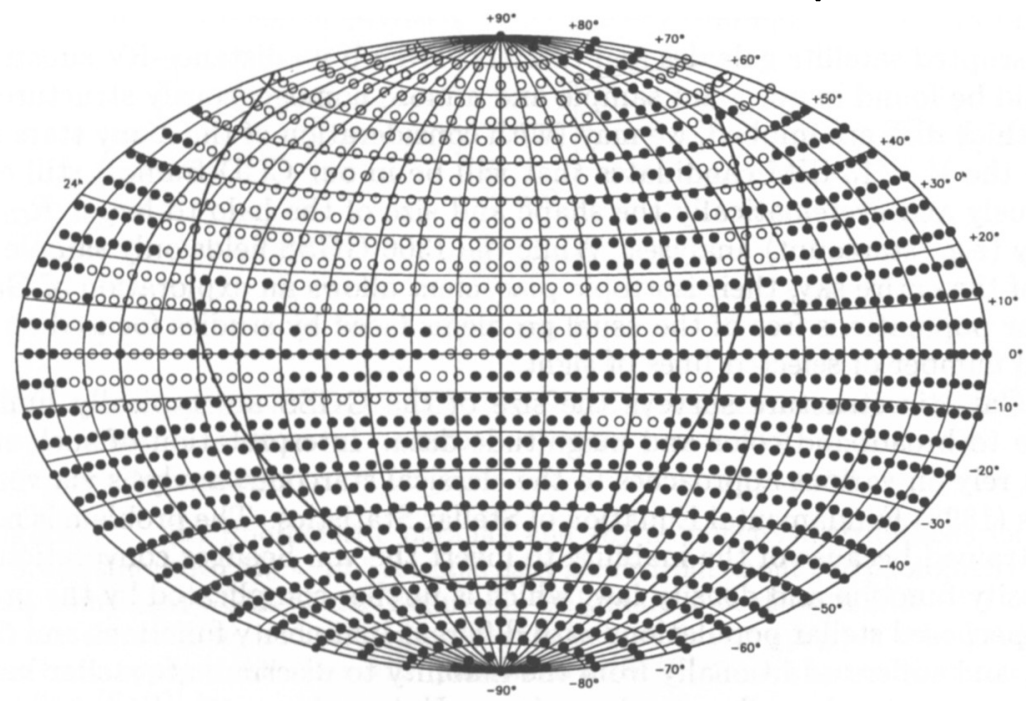

Figure 3. Aitoff projection in equatorial coordinates showing the distribution of GGSS fields to be observed. Filled circles indicate fields for which photometric data has been obtained as of 1 Feb 2001. The Galactic Equator is shown as the solid curve.

\subsection{High Resolution Spectroscopy}

The final phase of the project (being conducted in collaboration with several groups, including V. Smith using the McDonald 82-inch and D. Geisler using the FEROS on the ESO 1.52-m) involves (1) high resolution $(\sim 20 \mathrm{~m} / \mathrm{s}) \mathrm{spec}-$ troscopic radial velocity monitoring and (2) photometric monitoring (being conducted by Geisler with the Las Campanas 1-m and CTIO 0.9-m telescopes, and Smith with the McDonald 0.8-m). Both of these monitoring projects will help in the elimination of binaries and stars with large planets, as well as chromospherically active stars, from the SIM Grid. Frink et al. (2001) have found that the intrinsic radial velocity variations for single $\mathrm{K}$ giants peaks around $20 \mathrm{~m} / \mathrm{s}$; reflex velocity sensitivity at this level will allow $\mathrm{K}$ giants with stellar, brown dwarf (which are in any case expected to be rare due to the observed "Brown Dwarf Desert"; Marcy \& Butler 2000) and Jupiter-mass planetary companions with a semimajor axis $a \lessgtr 2$ AU to be identified, and left out of the SIM Grid. Lower mass planetary companions to distant sub-solar metallicity $\mathrm{K}$ giants will not affect the astrometric stability at the $4 \mu$ as level. Therefore, most companions that will affect the Grid can be ruled out with a moderate number $(\sim 5$ ) of radial velocity visits, and nearly all of the remaining contaminants can be ruled out with SIM itself. We expect a contamination level of undetectable astrometrically unstable stars in the Grid of less than $1 \%$.

\section{Characteristics of and Uses for Stars in the Survey}

We have already demonstrated (Majewski et al. 1999,2000b) how giants can be used to find and potentially map halo substructure in the form of tidal debris 
of disrupted satellite galaxies and clusters. Even if no distance-RV substructure should be found in our giant sample and a more homogeneously structured halo and thick disk are implied, we may (with consideration of the many stars fainter than the $V=13$ SIM candidates that will be in our CCD frames) still explore uniquely and systematically the shape and size of the halo to larger $R_{G C}$ than many (e.g., starcount) analyses. While the 1306 GGSS fields only sample about $4 \%$ of the entire sky, their isotropic placement allows an exploration of Galactic stellar populations free of the usual problems faced by studies focused on just a small number of selected lines of sight.

Galactic structure surveys the size of the GGSS are typically limited in scope to broadband color and magnitude data. Interpretation of such surveys must rely on various approaches to the classical starcount analysis via von Seeliger's (1898) Fundamental Equation of Stellar Statistics. The problem is not well constrained because of the inability to invert the von Seeliger convolution of luminosity function and density law, which is further complicated by the presence of superposed stellar populations with different luminosity functions and density laws, and suffers additionally from the inability to discriminate stellar luminosity classes using broadband colors alone. Unless the survey keys on a rather special, and readily identifiable, class of star (e.g., RR Lyrae stars, Cepheids), considerable effort must be expended to ascertain the luminosity class of the sample stars (e.g., through spectroscopy or trigonometric parallaxes). In large or faint surveys for which such effort presents a daunting challenge, it is often simpler to assume a luminosity class for the stars under study and recognize (or model) the expected systematic errors on the survey results. For example, universally asserting that all stars are dwarfs introduces a systematic underestimate of distances and transverse velocities, since some stars will be giants or subgiants.

An advantage of the GGSS survey is that not only does our photometry yield the usual colors and magnitudes of a large area photometric survey, but the inclusion of the $D D O 51$ intermediate band filter provides us with unprecedented leverage on luminosity classes for stars in such a huge dataset: We can rather reliably separate late type dwarfs from evolved stars on the red giant branch, breaking a particularly troublesome color-magnitude degeneracy. This will allow us to obtain photometric parallaxes for individual stars directly. Thus, we avoid the usual modus operandi of treating this problem in a statistical way across the survey.

Surface gravity alone is insufficient to determine precisely the intrinsic luminosity of a star; both metallicity and surface gravity determine the absolute magnitude of a star of given surface temperature. Fortunately, our filter system provides some additional sensitivity to a wide range of abundances in late $\mathrm{G}$ to early $M$ giants (see Figure 2). The combined gravity and abundance data improve the accuracy of our photometric parallaxes, as the giant branch luminosities at a given color vary by as much as 3 magnitudes over the range $-2<$ $[\mathrm{Fe} / \mathrm{H}]<0$.

A primary goal of SIM is to understand the size and structure of the dark matter halo of the Milky Way. Our fainter (i.e., $V=13-17$ ), halo giants from the GGSS are primary targets for just such a program because they are both relatively distant, but bright. Stars that can be verified as members of coherent 

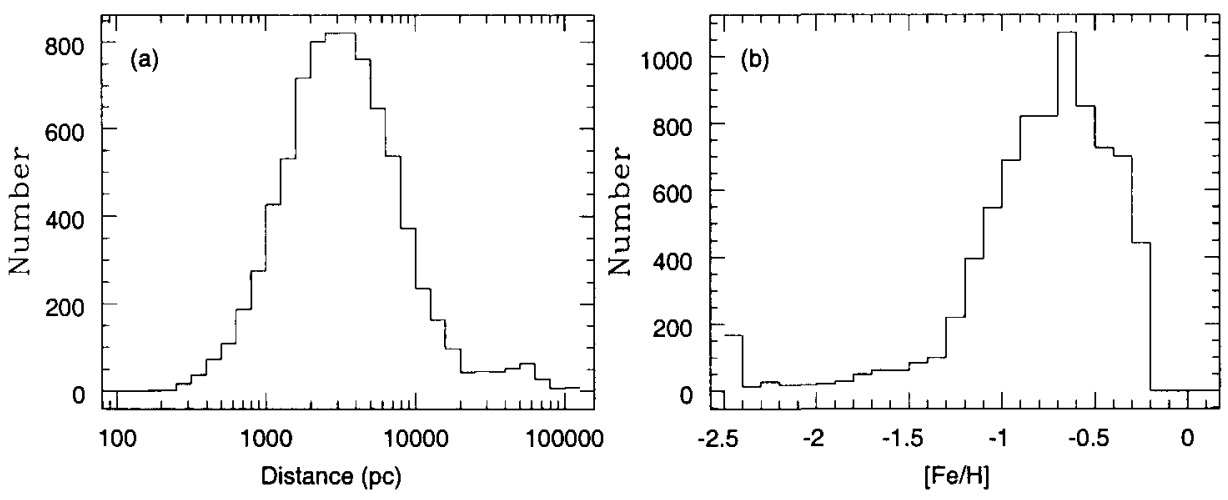

Figure 4. (a) Photometric parallax and (b) metallicity distributions for $\sim 8,000$ stars with $M_{o}<15.5$ selected as $\mathrm{K}$ giants from about 580 photometrically completed GGSS bricks (Figure 3). Only stars in fields with $E(B-V)<$ 0.2 are shown here, and photometric error cuts of $\left(\sigma_{M}, \sigma_{T}, \sigma_{D}\right)<0.04$ have also been applied. All stars with photometric $[\mathrm{Fe} / \mathrm{H}]<-2.5$ are combined in the first $[\mathrm{Fe} / \mathrm{H}]$ bin.

tidal structures have increased value for this work; Johnston et al. (1999) discuss an algorithm that can recover the circular velocity and flattening of the Milky Way to within $2.5 \%$ using SIM measurements of just 100 stars known to be members of a tidal tail associated with any one of the Galactic satellites. In order to understand the mass distribution of the inner Galaxy and its disk, SIM will need to measure parallaxes and proper motions for stars to at least $20 \mathrm{kpc}$ in all directions as a means to undertake definitively both the classical $K_{z}$ problem - determining the dependence of the Galactic potential on distance from the Galactic plane - as well as for measuring the Galactic rotational velocity to $2 R_{o}$. The GGSS will provide several times more than the number of giant stars in the desired range of distances (to $20 \mathrm{kpc}$ ) required for such studies (Figure 4a). Finally, the upcoming availability of the unique GGSS dataset has already spawned a new study into the binary and variable fraction of Pop II stars by Smith, Geisler and collaborators, and is providing lists of bright, metal-poor stars (see Figure $4 \mathrm{~b}$ ) that are valuable targets for high resolution spectroscopic studies of chemical abundances in the early Galaxy.

Acknowledgments. It is a pleasure to thank Steven Unwin and Ann Wehrle of JPL for their assistance in setting up this project. This work has been supported by NASA/JPL grants 1201670 and 1222563, a David and Lucille Packard Foundation Fellowship, and a Cottrell Scholar Award from The Research Corporation.

\section{References}

Cameron, A. G. W. 1988, ARA\&A, 26, 441

Canterna, R. 1976, AJ, 81, 228

Carney, B. W., Laird, J. B., Latham, D. W. \& Aguilar, L. A. 1996, AJ, 112, 668

Cuntz, M., Rammacher, W. \& Ulmschneider, P. 1994, ApJ, 432, 690 
Duquennoy, A. \& Mayor, M. 1991, A\&A, 248, 485

Dupree, A. K. \& Smith, G. H. 1995, AJ, 110, 405

Frink, S., Quirrenbach, A., Fischer, D., Röser, S. \& Schilbach, E. 2001, PASP, 113,173

Geisler, D. 1984, PASP, 96, 723

Gilliland, R. L. et al. 2000, ApJ, 545, L47

Gonzalez, G. 1999, MNRAS, 308, 447

Gray, D. F. 1989, ApJ, 347, 1021

Hall, D. 1991, in IAU Colloquium 130, eds. I. Tuominen, D. Moss \& G. Rüdiger, (New York: Springer), p 353

Harris, H. C. \& McClure, R. D. 1983, ApJ, 265, L77

Hut, P. et al. 1992, PASP, 104, 981

Johnston, K. V., Zhao, H., Spergel, D., \& Hernquist, L. 1999b, ApJ, 512, L109

Majewski, S. R., Ostheimer, J. C., Kunkel, W. E. Johnston, K. V., Patterson, R. J. \& Palma, C. 1999, IAU Symp. 190, 508

Majewski, S. R., Ostheimer, J. C., Kunkel, W. E. \& Patterson, R. J. 2000a, AJ, 120,2550

Majewski, S. R., Ostheimer, J. C., Patterson, R. J., Kunkel, W. E., Johnston, K. V. \& Geisler, D. 2000b, AJ, 119, 760

Marcy, G. W. \& Butler, R. P. 2000, PASP, 112, 137

Nissen, P. A. \& Schuster, W. J. 1991, A\&A, 251, 457

Rutten R. G. M., Schrijver, C. J., Lemmens, A. F. P., \& Zwaan C. 1991, A\&A, 252,203

Ryan, S. G. 1992, AJ, 104, 1144

Simon, T. \& Drake, S. A. 1989, ApJ, 346, 303

Stevenson, D. J. 1985, Icarus, 62, 4

von Seeliger, H. H. 1898, in Papers of the Mathematical-Physics Class of the Bavarian Academy of Sciences, 19, 565 